\title{
Tumor invasion: molecular shears blunted by green tea
}

To the editor-The recent press, both popular and scientific, has given wide coverage of the beneficial properties of green tea, most commonly used in Asian countries. Consumption has been associated with prevention of cancer development and metastasis ${ }^{1}$. The main flavonol of green tea, epigallocatechin3-gallate (EGCG), inhibits urokinase ${ }^{2}$, one of the hydrolases implicated in tumor invasion. Moreover, green tea consumption by mice significantly limits angiogenesis ${ }^{3}$, crucial for the growth of all solid tumors.

Tumor invasion and angiogenesis are reduced, although with uncomfortable side effects, by the administration of synthetic inhibitors of matrix metalloproteinases (MMPs). Our findings show that micromolar EGCG inhibits tumor cell invasion and directly suppresses the activity of MMP-2 and MMP-9, two of the proteases most frequently overexpressed in cancer and angiogenesis, and essential in cutting through basement membrane barriers ${ }^{4}$.

The research on urokinase shows that EGCG directly impairs a molecular mechanism active in degradation of extracellular matrix. We would go further, and suggest that the activity of the green tea component on urokinase may inhibit the enzymatic cascade ending in activation of gelatinases MMP-2 and MMP-9; however, the effective EGCG concentration has been considered to far exceed the levels found in vivo ${ }^{3}$. At
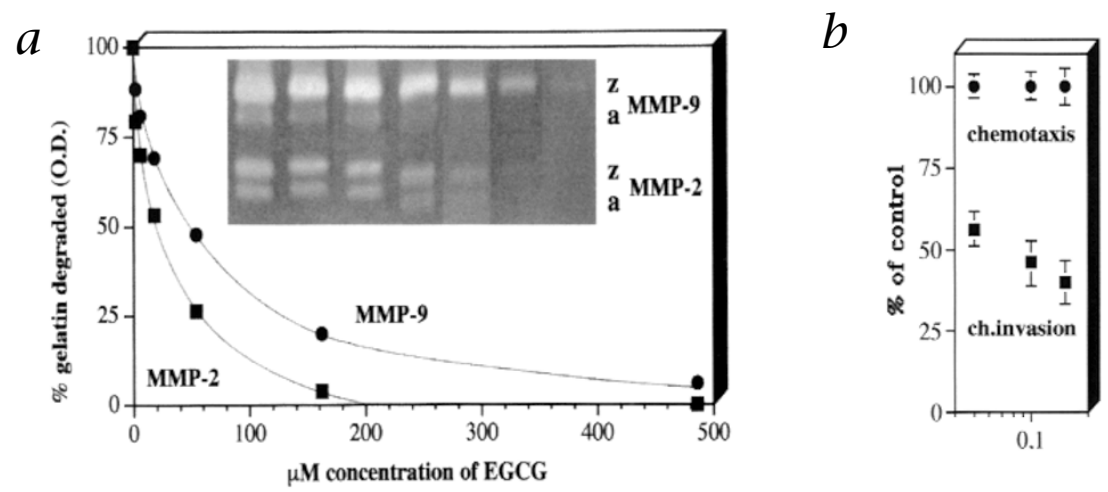

Fig. 1 a, Zymographic evidence of direct suppression of MMP. Serum-free DMEM conditioned by $5 \times 10^{4} \mathrm{HT} 1080$ fibrosarcoma cells was separated by electrophoresis in $0.1 \%$ gelatin-containing polyacrylamide. After removal of SDS, separate lanes were incubated overnight at $37^{\circ} \mathrm{C}$ in Tris$\mathrm{CaCl}_{2}$ buffer, $\mathrm{pH} 7.4$, in presence of increasing concentrations of EGCG; the $\mathrm{pH}$ of the buffer, was unaltered even at highest concentration. Inset, Coomassie blue staining. Densitometric values of digestion bands were plotted for zymogen ( $\mathrm{z}$ ) and activated (a) forms of each gelatinase. $\boldsymbol{b}, \mathrm{HT} 1080$ cells (expressed as \% of control) recovered after $5 \mathrm{~h}$ onto the bottom surface of the Boyden chamber filter after traversing gelatin (chemotaxis) and Matrigel ${ }^{R}$ (chemoinvasion) coatings, towards a chemoattractant. s.e.m. of triplicate experiments. parent contradiction: certain MMPs, such as MMP-3, -7, -9 and -12, in addition to their essential role in tumor and endothelial cell invasion, can also contribute to the generation of angiostatin, a potent angiogenesis inhibitor derived by cleavage of plasminogen. Our results indicate that EGCG, as direct inhibitor of MMPs, inhibits angiogenesis and metastasis upstream of the action of angiogenesis inhibitors derived from the action of MMPs.

Our data show that the EGCG concentration effective in inhibiting MMP2 and MMP-9 is orders of magnitude lower than that reported for urokinase (down to 1/500), and that an even lower concentration (equivalent to that in the plasma of drinkers of moderate amounts of green tea) is effective in reducing tumor cell invasion by $50 \%$. We suggest that green tea exerts its beneficial effects against cancer by impairing tumor invasion and nourishment through direct inhibition of two gelatinases that open up pathways for cell migration. Furthermore, in addition to having a preventive role, EGCG may be effective in combination with angiostatin (inhibitor of endothelial cell proliferation) in a dual-action clinical treatment.

Spiridione Garbisa ${ }^{1}$, Susan BigGin ${ }^{2}$, Nadia Cavallarin ${ }^{1}$, LUigi Sartor ${ }^{1}$, Roberto BENELli ${ }^{3} \&$ Adriana AlBINI ${ }^{4}$ ${ }^{1}$ Institute of Histology and Embryology,

Medical School

35121 Padova, Italy

${ }^{2}$ UNIDO-ICS

AREA Science Park

34012 Trieste, Italy

${ }^{3}$ Tumor Progression Section and

${ }^{4}$ Molecular Biology Laboratory

National Institute for Research on Cancer

IST, c/o CBA

Genova, Italy

Email: garbisa@civ.bio.unipd.it

1. Taniguchi, S. et al. Effect of (-)-epigallocatechin gallate, the main constituent of green tea, on lung metastasis with mouse B16 melanoma cell lines. Cancer Lett. 65, 51-54 (1992).

2. Jankun, J., Selman, S.H. \& Swiercz, R., SkrzypczakJankun, E. Why drinking green tea could prevent cancer. Nature 387, 561 (1997).

3. Cao, Y. \& Cao, R. Angiogenesis inhibited by drinking tea. Nature 398, 381 (1999).

4. Liotta, L.A. et al. Metastatic potential correlates with enzymatic degradation of basement membrane collagen. Nature 284, 67-68 (1980).

5. Albini, A. et al. A rapid in vitro assay for quantitating the invasive potential of tumor cells. Cancer Res. 47, 3239-3245 (1987). 